\title{
Numerical modelling of the filling of formworks with self-compacting concrete
}

\author{
S. Tichko ${ }^{1}$, J. Van De Maele ${ }^{2}$, N. Vanmassenhove ${ }^{2}$, \\ G. De Schutter ${ }^{1}$, J. Vierendeels ${ }^{3}$, R. Verhoeven ${ }^{4} \&$ P. Troch ${ }^{5}$ \\ ${ }^{1}$ Magnel Laboratory for Concrete Research, Ghent University, Belgium \\ ${ }^{2}$ Ghent University, Belgium \\ ${ }^{3}$ Dept. of Flow, Heat \& Combustion Mechanics, Ghent University, \\ Belgium \\ ${ }^{4}$ Laboratory for Hydraulics, Ghent University, Belgium \\ ${ }^{5}$ Department of Civil Engineering, Ghent University, Belgium
}

\begin{abstract}
This paper describes the numerical modelling of the flow of self-compacting concrete (SCC) in column and wall formworks during the filling process. It is subdivided into four main parts. In the first part, the rheological properties of SCC and the theory regarding the pressure exerted by the SCC on the formworks are shortly described. In the second part, the formwork filling tests, which have been carried out at the Magnel Laboratory for Concrete Research of the Ghent University, are presented. The general layout of the tests and the measurement set-up are clearly described. In the third part, the numerical modelling of the flow of SCC using a commercially available solver is explained as well as the obtained results from the CFD simulations. Finally in the last part, a comparison is made between the measurements and the simulation results. The formwork pressures are hydrostatic for SCC pumped from the base of the formworks. Keywords: CFD, numerical modelling, laminar flow, self-compacting concrete.
\end{abstract}

\section{Rheology of SCC and the theory of formwork pressure}

\subsection{Rheology of SCC}

According to De Schutter et al [1], SCC can be defined as a concrete which needs to possess sufficient fluidity in order to be able to fill a formwork 
completely (filling ability) without the aid of other forces than gravity, even when having to flow through narrow gaps (passing ability), but also showing a sufficient resistance to segregation, during flow and in stationary conditions (stability).

In order to achieve sufficient fluidity in SCC, without increasing the water content, super-plasticizers must be applied. Only adding super-plasticizers to traditional concrete is not sufficient to create SCC, due to the large amount of coarse aggregates, which can form particle bridges when flowing through a narrow gap, causing blocking. Therefore in order to fulfil the passing ability condition, the amount of coarse aggregates is reduced. On the other hand, extra amounts of fine materials, like limestone filler, fly ash, silica fume are added in order to increase the stability of SCC $[1,2]$.

According to Feys [2] and Roussel and Lemaitre [2, 3], the behaviour of fresh concrete may be described using a yield stress model such as the Bingham model or the Herschel-Bulkley model, which are formulated mathematically by eqn (1) and eqn (2) respectively.

$$
\begin{gathered}
\tau_{B}=\tau_{0, B}+\mu_{p, B} \cdot \dot{\gamma} \\
\tau_{H B}=\tau_{0, H B}+K_{H B} \cdot \dot{\gamma}^{n_{H B}}
\end{gathered}
$$

where: $\quad$ the indices B, HB stand for Bingham, Herschel-Bulkley, resp.

$\tau \quad=$ shear stress in the material $(\mathrm{Pa})$

$\gamma \quad=$ shear rate in the material $(1 / \mathrm{s})$

$\tau_{0} \quad=$ yield stress $(\mathrm{Pa})$

$\mu_{p, B}=$ plastic viscosity (Pa s)

$K_{H B}=$ consistency factor $\left(\mathrm{Pa} \mathrm{s}^{n}\right)$

$n_{H B}=$ consistency index (-)

SCC is further characterized by two specific phenomena, thixotropy and shear thickening. Thixotropy can be defined as a reversible build-up and breakdown of internal structure due to flocculation or coagulation of cement particles for which the influence of inter-particle forces is still significant, whereas shear thickening is an increase in apparent viscosity with increasing shear rate.

In order to capture the effect of shear thickening in SCC, Feys [2] proposed a modification of the standard Bingham material model. This modified Bingham model is defined by the following equation.

$$
\tau_{M B}=\tau_{0, M B}+\mu_{p, M B} \cdot \dot{\gamma}+c_{M B} \cdot \dot{\gamma}^{2}
$$

where: $\quad$ the indices MB stands for modified Bingham

$$
c_{M B}=\text { second order parameter }\left(\mathrm{Pa} \mathrm{s}^{2}\right)
$$


The effect of shear thickening becomes very important at high shear rates. This is captured in the modified Bingham model by the extra quadratic term in eqn (3).

Roussel [4] proposed the following model for describing thixotropy in SCC. He introduced a structure parameter $\lambda$, the flocculation state of the material, which influences the apparent rheological properties of the material. It is defined by two equations, where three parameters have to be identified.

$$
\begin{gathered}
\tau=(1+\lambda) \cdot \tau_{0, B}+\mu_{p, B} \cdot \dot{\gamma} \\
\frac{\partial \lambda}{\partial t}=\frac{1}{T}-\alpha \cdot \lambda \cdot \dot{\gamma}
\end{gathered}
$$

where: $\quad \lambda=$ the flocculation state of the material (-)

$T=$ flocculation characteristic time (s)

$\alpha=$ de-flocculation parameter $(-)$

Eqn (4) is a constitutive equation and eqn (5) describes the rate of change of the internal structure.

It is assumed that the characteristic time of flocculation is long compared to the characteristic time of de-flocculation [5]. Eqn (5) then simplifies to:

$$
\frac{\partial \lambda}{\partial t}=-\alpha \cdot \lambda \cdot \gamma
$$

After integration of eqn (6), and introducing the resulting flocculation state in eqn (4), the shear stress then writes:

$$
\tau=\left(1+\lambda_{0} \cdot e^{-\alpha \dot{\gamma} t}\right) \cdot \tau_{0, B}+\mu_{p, B} \cdot \dot{\gamma}
$$

At rest, the shear rate equals zero and the evolution of the apparent yield stress is described by eqn (8).

$$
\tau=\tau_{0}(t)=(1+\lambda) \cdot \tau_{0}=\tau_{0}+\tau_{0} \cdot \frac{t}{T}=\tau_{0}+A_{t h i x} . t
$$

Based on the experience of Roussel [4] with formwork pressure measurements, a classification of SCC according to their flocculation rate $\mathrm{A}_{\text {thix }}$ can be made. This classification is presented in Table 1 . 
Table 1: $\quad$ Classification of SCC according to their flocculation rate.

\begin{tabular}{|c|c|}
\hline Flocculation rate $\mathbf{A}_{\text {thix }}(\mathbf{P a} / \mathbf{s})$ & SCC type \\
\hline Less than 0,1 & Non-thixotropic SCC \\
\hline Between 0,1 and 0,5 & Thixotropic SCC \\
\hline Higher than 0,5 & Highly thixotropic SCC \\
\hline
\end{tabular}

\subsection{Theory of formwork pressure}

In practice, two types of filling processes are encountered, namely filling from the top of the formwork and filling from the base of the formwork. When the formwork is filled from the top, due to thixotropy the SCC starts to build up a structure able to withstand pressure from the concrete above, without increasing the horizontal pressure against the form [5]. For this type of formwork filling, the horizontal formwork pressure can be calculated using eqn (9) [4].

$$
p_{\text {hor }}=\left(1-\frac{A_{\text {thix }} \cdot H}{\rho_{S C C} \cdot g \cdot R \cdot e}\right) \rho_{S C C} \cdot g \cdot H
$$

$$
\text { where: } \quad \begin{array}{ll}
e & =\text { the casted wall thickness }(\mathrm{m}) \\
H & =\text { height of the casted element }(\mathrm{m}) \\
\rho_{S C C} & =\text { density of the } \operatorname{SCC}\left(\mathrm{kg} / \mathrm{m}^{3}\right) \\
g & =\text { gravity acceleration }\left(\mathrm{m} / \mathrm{s}^{2}\right) \\
R & =\text { casting rate }(\mathrm{m} / \mathrm{s})
\end{array}
$$

For the case where the SCC is pumped from the base of the formwork, the formwork pressure is mainly hydrostatic [5]. The reason is because the SCC is in motion during the whole casting procedure, without any possibility for the concrete shear strength to develop. The resulting formwork pressure can then be determined using eqn (10).

$$
p_{\text {hor }}=\rho_{S C C} \cdot g \cdot H
$$

\section{Full-scale formwork filling tests}

In this part, the four full-scale formwork filling tests that have been performed by Van De Maele and Vanmassenhove [6] at the Magnel Laboratory for Concrete Research of the Ghent University in Belgium are described. During these tests, two columns and two walls were cast, as shown in Fig. 1.

The two columns are identical and have a height of $2 \mathrm{~m}$, a depth of $21 \mathrm{~cm}$ and a width of $17,4 \mathrm{~cm}$. The SCC is pumped from the base of the formwork. The two walls are also identical regarding the dimension (a height of $2 \mathrm{~m}$, a length of $4 \mathrm{~m}$ and a thickness of $21 \mathrm{~cm}$ ), but not regarding the positioning of the SCC inlet. 


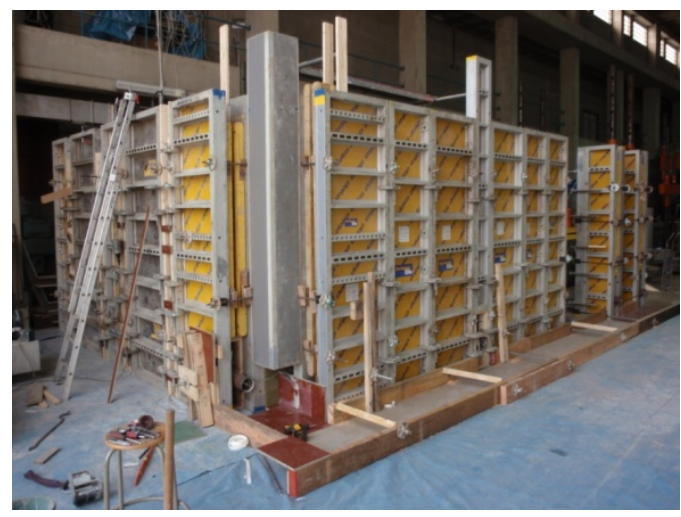

Figure 1: $\quad$ Layout of the formwork filling tests.

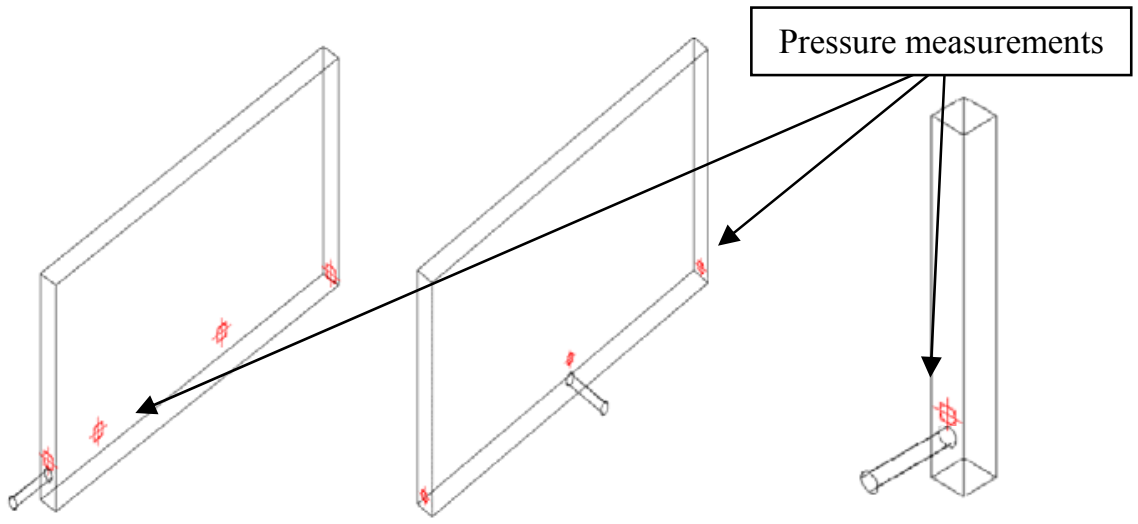

Figure 2: $\quad$ Connections of the SCC inlet for wall type A and B.

Fig. 2 shows the two different pumping connections. For both walls, the SCC is also pumped from the base of the formwork.

During the filling of the formworks, the flow of the SCC was measured at the concrete pump, the time was recorded and the progression of the filling process was monitored with several cameras. The formwork pressure was also measured on several positions at the base of the formwork, where the maximum pressure was expected. Two types of pressure measurements were used: manometers and electronic pressure transducers. Both devices were fixed on an intermediate water chamber, sealed with a rubber membrane, and flush mounted on the formwork. The locations of the pressure measurements (red cross-haired squares) are shown on fig. 2 for the walls and for the columns. At the beginning of each filling process and at the end of the casting test period, a sample of concrete has been taken in order to investigate the fresh properties, by means of a Tattersall Mk-II rheometer and standard tests on SCC like slump flow, V-funnel, L-box, sieve stability and air content. 


\section{Numerical modelling of the formwork filling tests}

\subsection{Governing equations}

The most general description of a fluid flow is obtained from the well known Navier-Stokes equations. They represent mass conservation, conservation of motion and conservation of energy [7,8]. Due to fact that the density and the viscosity of the SCC remains constant during the isothermal filling process, the conservation of energy is not considered in the numerical simulations. For unsteady, isothermal, laminar flows these equations take on the following forms:

Conservation of mass:

$$
\frac{\partial \rho}{\partial t}+\nabla(\rho \cdot \vec{v})=0
$$

Conservation of momentum:

$$
\frac{\partial(\rho \cdot \vec{v})}{\partial t}+\nabla(\rho \cdot \vec{v} \otimes \vec{v})=-\nabla p+\nabla \bar{\tau}+\rho \cdot \vec{g}
$$

where: $\quad \rho \quad=$ density or specific mass of the fluid $\left(\mathrm{kg} / \mathrm{m}^{3}\right)$

$\vec{v} \quad=$ velocity vector $(\mathrm{m} / \mathrm{s})$

$\bar{\tau} \quad=$ stress tensor $(\mathrm{Pa})$

$\vec{g} \quad=$ gravity acceleration vector $\left(\mathrm{m} / \mathrm{s}^{2}\right)$

$\otimes \quad=$ tensor product of two vectors

Due to the fact that the shear rates remain relatively low during the formwork filling process, no shear thickening is occurring. Therefore the stress tensor $\tau$ is modelled using the Herschel-Bulkley material model (see also eqn (2)). These Navier-Stokes equations are solved for the pressures and the velocities over the entire computational domain for each time step with the aid of the Fluent v.6.3 software. In order to simulate a free surface flow, as in the formwork filling process, the Volume of Fluid (VOF) method developed by Hirt and Nichols [9], is used. The VOF method can model two or more immiscible fluids by solving a single set of momentum equations and tracking the volume fraction of each of the fluids throughout the domain.

\subsection{Computational domain}

In order to perform the numerical simulation of the SCC flow through the formworks shown in fig. 1, a 3D geometry is constructed for each cast element and meshed with the Fluent Gambit v.2.3.16 pre-processor [10]. Fig. 3, fig. 4 and fig. 5 show the resulting high quality meshes for the columns and the walls. 


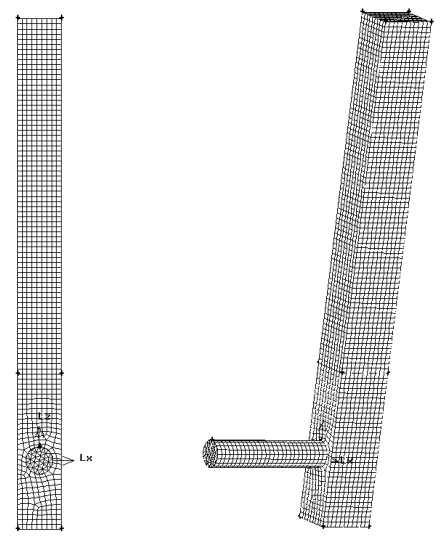

Figure 3: Computational domain for the column filling process.

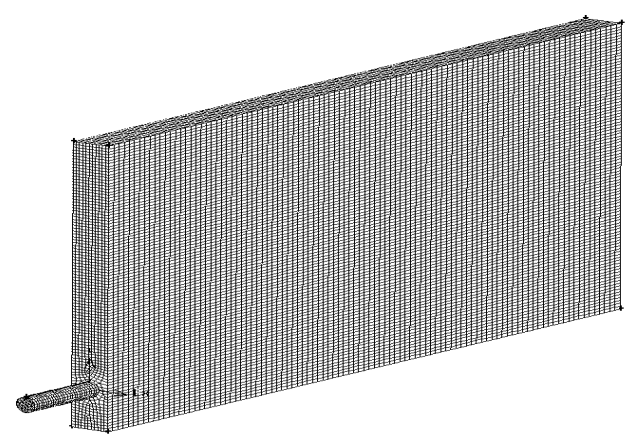

Figure 4: Computational domain for wall type A filling process.

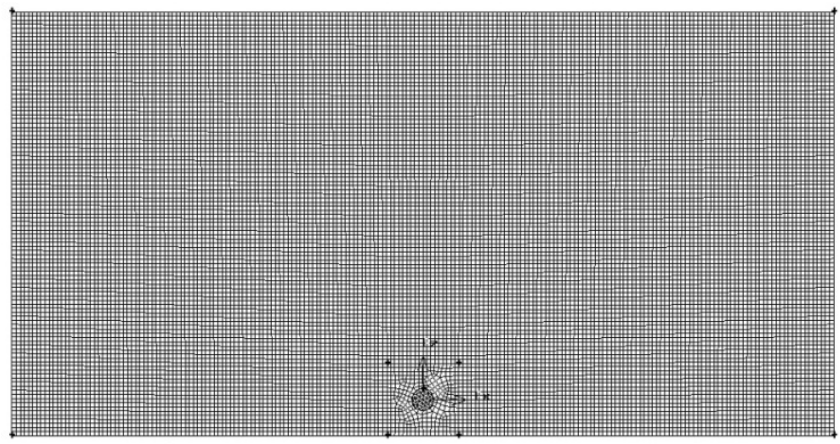

Figure 5: Computational domain for wall type B filling process. 


\subsection{Boundary conditions}

In order to obtain solutions for the stated Navier-Stokes equations, a set of boundary conditions needs to be applied to the computational domain. At the flow inlet, a uniform velocity profile of the incoming SCC and the volume fraction of the SCC phase is imposed. At the top of the formwork, the pressure is atmospheric, resulting in a zero gauge pressure condition at the outlet. At the pipe walls, a no-slip condition is assumed.

\subsection{Solution of the governing equations}

The governing flow equations are discretized using the Finite Volume Method and solved with a 3D, double precision, implicit pressure-based solver [8]. In table 2 the solver settings of the numerical model are summarized.

Table 2: $\quad$ Solver controls.

\begin{tabular}{|c|c|c|}
\hline Controls & Columns & Wall type A and B \\
\hline Multiphase VOF model/scheme & 2 phases & 2 phases \\
\hline VOF scheme & Explicit & Explicit \\
\hline Transient controls & NITA $^{1}$ & NITA $^{1}$ \\
\hline Unsteady formulation & $1^{\text {st }}$ order implicit & $1^{\text {st }}$ order implicit \\
\hline Body force formulation & Implicit body force $_{\text {Implicit body force }}$ \\
\hline Momentum discretization & $2^{\text {nd }}$ order upwind & $2^{\text {nd }}$ order upwind \\
\hline Volume fraction discretization & Geo-reconstruct & CICSAM $^{2}$ \\
\hline Pressure discretization & Body force weighted & Body force weighted \\
\hline Pressure-velocity coupling & Fractional step method & Fractional step method \\
\hline
\end{tabular}

${ }^{1}$ Non-Iterative Time Advancement

${ }^{2}$ Compressive Interface Capturing Scheme for Arbitrary Meshes

\subsection{Formwork filling simulations}

The fresh properties of the SCC used in the formwork filling tests are the presented in table 3 .

Table 3: $\quad$ Fresh properties of the SCC used in the tests.

\begin{tabular}{|c|c|}
\hline Property & Value \\
\hline Density $\rho_{\mathrm{SCC}}\left(\mathrm{kg} / \mathrm{m}^{3}\right)$ & 2314,4 \\
\hline Viscosity $\mu_{\mathrm{p}, \mathrm{HB}}(\mathrm{Pa} \mathrm{s})$ & 28,3 \\
\hline Yield stress $\tau_{0, \mathrm{HB}}(\mathrm{Pa})$ & 10,3 \\
\hline Consistency index $\mathrm{n}_{\mathrm{HB}}(-)$ & 1,385 \\
\hline Consistency factor $\mathrm{K}_{\mathrm{HB}}\left(\mathrm{Pa} \mathrm{s}^{\mathrm{n}}\right)$ & 17,7 \\
\hline
\end{tabular}

Fig. 6 show the simulated formwork filling of the columns, while the simulated casting process of the walls are presented in fig. 7 and fig. 8 . 


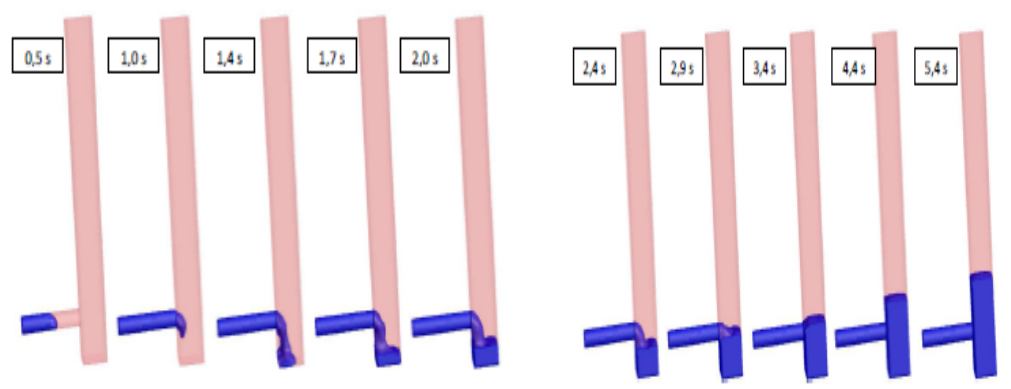

Figure 6: Simulated filling process of the columns.
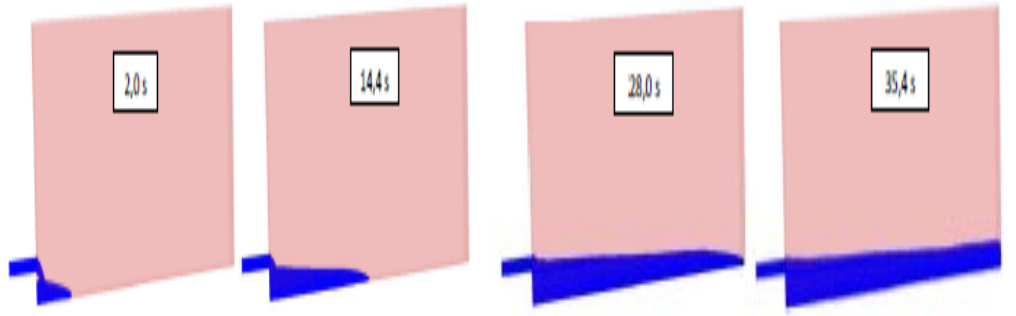

Figure 7: $\quad$ Simulated filling process of wall type A.
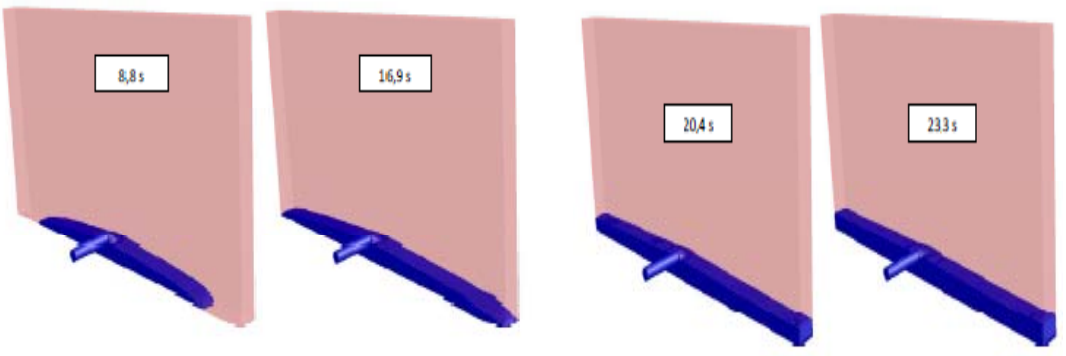

Figure 8: $\quad$ Simulated filling process of wall type B.

\section{Comparison of the results and conclusions}

\subsection{Comparison of the results}

Without the presence of thixotropic build-up, the formwork pressures are expected to be hydrostatic, and therefore they can be calculated analytically using eqn (10). The measured formwork pressures are compared with the hydrostatic pressures and presented in fig. 9 for the columns and in fig. 10 for the two wall types. 


\section{Column formwork pressure}

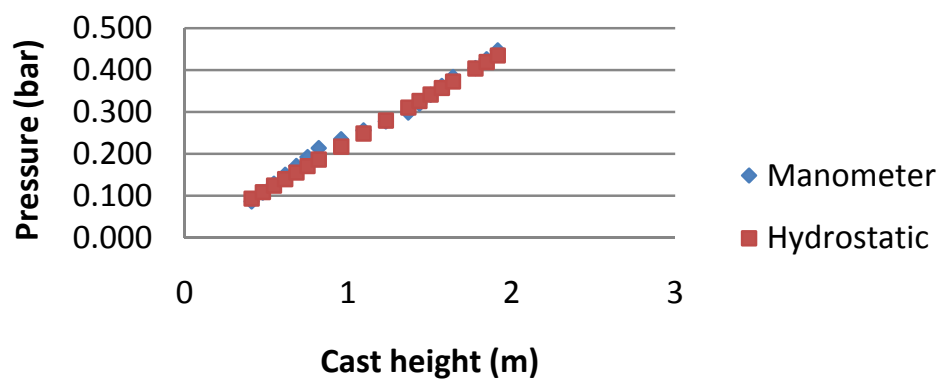

Figure 9: $\quad$ Formwork pressure of the columns.

Wall type A and B formwork pressures

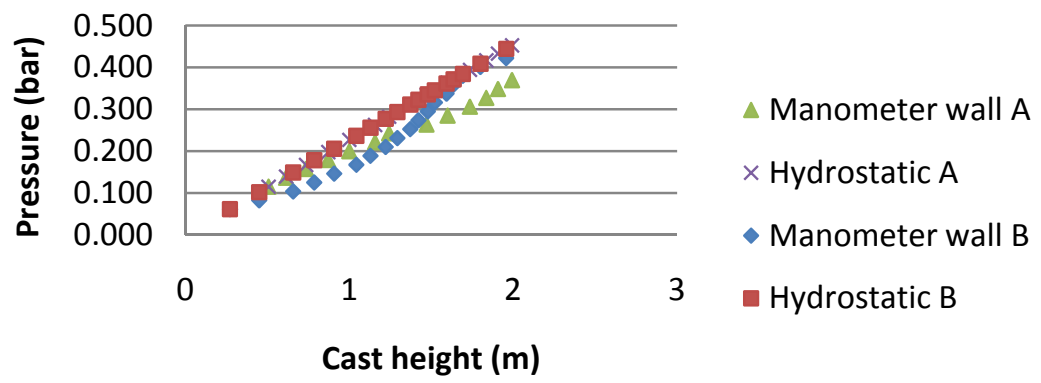

Figure 10: $\quad$ Formwork pressure of the wall type A and B.

The formwork pressures obtained from the numerical simulations are shown in fig. 11 for the columns and in fig. 12 for the two wall types.

\section{Column formwork pressure}

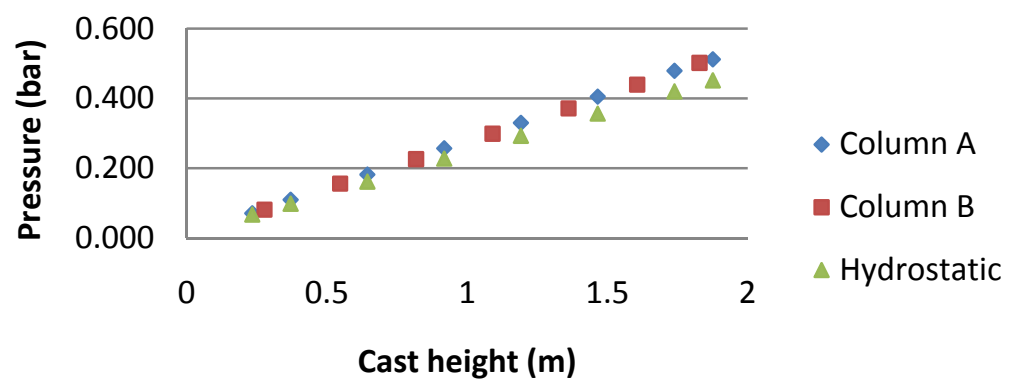

Figure 11: Column formwork pressure from CFD simulation. 


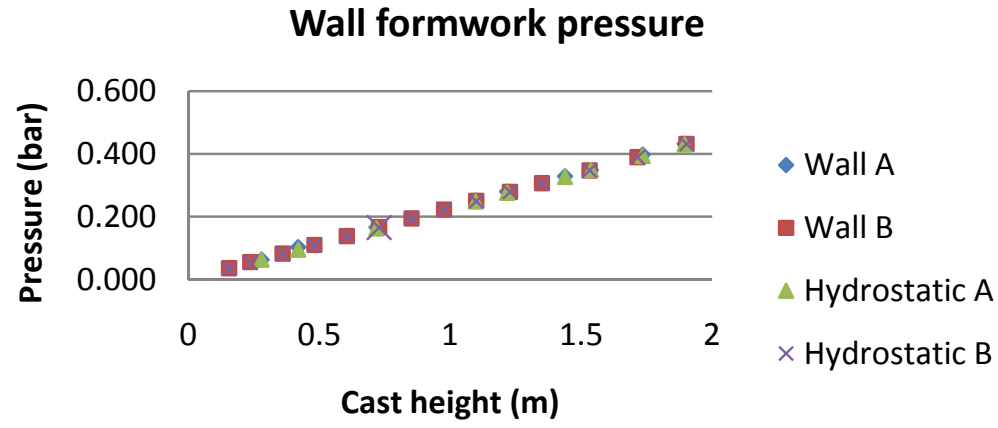

Figure 12: Wall formwork pressure from CFD simulations.

\subsection{Conclusions}

From the comparison of the measurements, the analytical results and the results from the CFD simulations, we can conclude that the formwork pressures are hydrostatic as expected [2]. The small differences that can be observed between the measured pressures and the hydrostatic pressures for the same cast heights is related to the experienced shortcomings of the pressure measurement units used in the tests. The non-linear deformation of the plastic membranes due to exerted concrete pressure, the variable surface tension of the used membranes and the unavoidable inclusion of air bubbles into the water chambers have lead to these small differences in the formwork pressure measurements.

Nevertheless, it can be concluded that there is a very good agreement between all the obtained results. The VOF method has proved to very effective in capturing the time evolution of a formwork filling process, as shown in fig. 13.
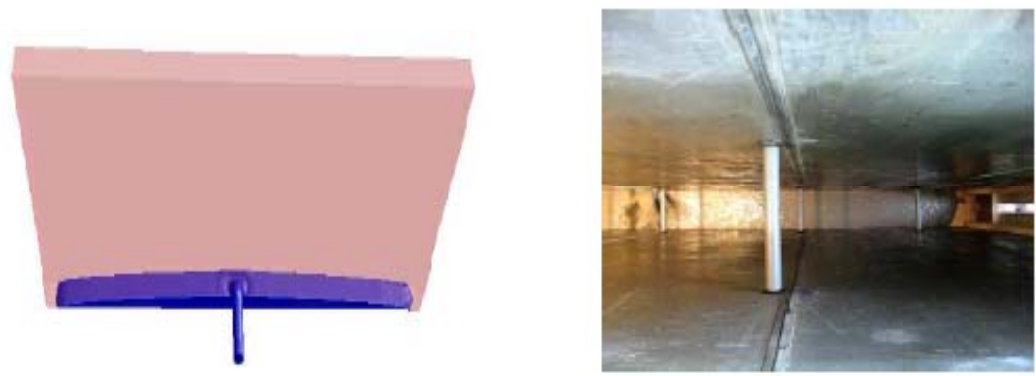

Figure 13: Simulated and real formwork filling evolution.

The real position and form of the free surface of the SCC, during the filling of the formwork of wall type $\mathrm{B}$, is compared with the simulated position and form of the SCC-air interface at the same time. 
For this type of casting processes, where the SCC is pumped from the base of the formwork to the top, as such avoiding thixotropic build-up, the formwork pressures can easily be estimated using the simple formula for the hydrostatic pressure, eqn (10). When more details on the formwork filling process are required, numerical simulations can be carried out using the implemented Herschel-Bulkley material model, describing the relation between the applied shear rate and the resulting shear stress in the SCC, in combination with the VOF method. These CFD simulations will predict the formwork pressures and the time evolution of the casting process accurately.

\section{References}

[1] De Schutter, G. and Bartos, P. and Domone, P. and Gibbs, J., SelfCompacting Concrete, Whittles Publishing, Caithness, 2008.

[2] Feys, D., Interactions between Rheological Properties and Pumping of Self-Compacting Concrete, Ph-D dissertation, Ghent University, 2009.

[3] Roussel, N. and Lemaître, A., Steady state flow of cement suspensions, Cem. Conc. Res., vol. 40, pp. 77-84, 2010.

[4] Roussel, N., A thixotropy model for fresh fluid concretes, Cem. Conc. Res., vol. 36, pp. 1797-1806, 2006.

[5] Billberg, P., Form pressure generated by SCC - Influence of Thixotropy and Structural Behaviour at Rest, Ph-D dissertation, Royal Institute of Technology, Stockholm, 2006.

[6] Van De Maele, J. and Vanmassenhove, N., Vullen van een bekisting met zelfverdichtend beton door het pompen van onderaan, Master thesis (in Dutch), Ghent University, 2010.

[7] Hirsch, Ch., Numerical Computations of Internal and External Flows, Volume 1 - Fundamentals of Numerical Discretization, John Wiley \& Sons, New York, 1989.

[8] Fluent 6.3 User's Guide, Fluent Inc, 2006.

[9] Hirt, C.W. and Nichols, B.D., Volume of Fluid Method for the Dynamics of Free Boundaries, J. Comput. Phys., vol. 39, pp. 201-225 1981.

[10] Gambit 2.3 Modelling Guide, Fluent Inc, 2006. 